NAN JIANG, Ph.D. Candidate ${ }^{1}$

(Corresponding author)

E-mail: jiangnantju@126.com

XIAONING ZHANG, Ph.D. ${ }^{2}$

E-mail: cexzhang@tongji.edu.cn

HUA WANG, Ph.D. ${ }^{2}$

E-mail: hwang63@tongji.edu.cn

${ }^{1}$ Key Laboratory of Road and Traffic Engineering of the

Ministry of Education, Tongji University

No.4800 CaoAn Road, Shanghai 201804, PR China

2 School of Economics and Management, Tongji University

No.1239 Siping Road, Shanghai 200092, PR China
Transportation Economy Original Scientific Paper Submitted: 9 Jan. 2017

Accepted: 3 July 2017

\title{
SIMULTANEOUS OPTIMIZATION OF ROAD TOLLS AND TRADABLE CREDITS IN PUBLIC-PRIVATE MIXED NETWORKS
}

\begin{abstract}
This paper investigates a hybrid management policy of road tolls and tradable credits in mixed road networks with both public and private roads. In the public sub-network, a tradable credit scheme is applied to mitigate traffic congestion. In the private sub-network, tolls are collected by the private company, but the toll levels and toll locations are determined by the government. The purpose of toll charge is two-fold: on the one hand, the government uses it as a tool for mitigating congestion; on the other hand, a threshold of revenue should be guaranteed for the profitability of the private company. A bi-level programming model is formulated to minimize the total travel time in the network by taking into account the user equilibrium travel behaviour and the revenue requirement of private firms. To obtain a global optimum solution, the bi-level model is transformed into an equivalent single-level mixed integer linear program that can be easily solved with commercial software. Numerical examples are provided to demonstrate the effectiveness of the developed model and the efficiency of the proposed algorithm. It is shown that the mixed management schemes can achieve favourable targets, namely, joint implementation of road tolls and tradable credits can effectively mitigate traffic congestion and meanwhile maintain reasonable revenue for the private company.
\end{abstract}

\section{KEY WORDS}

bi-level programming model; road tolls; tradable credits; mixed integer linear program; public-private mixed networks;

\section{INTRODUCTION}

In the past decades, substantial transportation infrastructure has been developed via private investment, especially in developing countries. For example, in China, transportation networks in several provinces have been substantially expanded in the form of private roads. Nevertheless, in both highway and urban road networks, public roads are still the major part of the transportation system. Therefore, a real network system often exists in the public-private mixed situation.

There are several management targets for the operation of the public-private mixed networks, due to the complicated benefit considerations of different stakeholders. First, with the steady growth of travel demand, transportation networks are often congested. Therefore the primal management goal of the government is to alleviate traffic congestion, especially in metropolitan areas. Second, for private firms, a reasonable level of revenue is necessary in order to ensure investment return, and to cover road maintenance cost.

Road pricing has long been recognized as an effective instrument for mitigating congestion and collecting revenue. The theoretical mechanism of road pricing is the principle of marginal cost pricing in economics that a traveller has to pay the cost they impose on others [1-5]. However, marginal cost pricing requires that tolls be charged on all links. Given the fact that it is almost impossible to collect tolls on all roads, many researchers turn to investigate second-best pricing schemes that only a subset of road links are charged, such as send-best link based tolls, cordon tolls, entry-exit based tolls and distance-based tolls [6-10]. On the other hand, tolls are also implemented on private roads in order to maintain a profitable situation of the private investors. For example, de Palma [11] developed a competition model based on simple networks with two parallel links, where each link was controlled by a private firm. Yang and Meng [12] considered the simultaneous determination of road tolls and link capacities on the private roads, while taking into account the network equilibrium. Yang and Woo [13] investigated the Nash equilibrium between two 
profit maximizing firms that controlled their own road links, respectively. Xiao et al. [14] analysed the toll and capacity competition among private asymmetric roads in a network with parallel links. Their results indicated that an oligopolistic competition generates higher toll and lower construction capacity than those in the case of social optimum. Wu et al. [15] demonstrated mathematically that in general road networks the service level provided by a profit-maximizing private firm was independent of another competitor's capacity and toll decision. Moreover, the volume versus capacity ratio remains the same even in a regulated market where the authority imposes a cap for the generalized travel cost on the private toll road.

Overall, congestion pricing is difficult to implement due to the objection of the public, since the governments are often criticized for collecting tolls on public roads. The good news is that researchers found new congestion mitigation methods, where travellers do not pay monetary cost to public authorities. For example, Yang and Wang [16], Xiao et al. [17] explored the mechanism of tradable mobility credits in transportation networks. In these policies, the government sets a goal and tries to achieve it by developing a market for free trading of mobility credits. Management authorities issue mobility credits to all eligible travellers, and determine how many credits are to be charged on each road. Once the management authority makes right decisions on credit provisions and link-based charges, the competitive market could spontaneously lead to a system optimum. The tradable credits scheme has some obvious advantages for the management of public roads. The scheme ensures high equity for the travellers as a public service, so it can avoid the political resistance due to the concern of unfairness. Most importantly, in the tradable credits scheme, the revenue-neutral attribution makes it more acceptable to the public. Recent development on the tradable credit in transportation network includes the work of Nie [18], where the effects of transaction costs in an artificial auction market and negotiated market were investigated. Wang et al. [19] proposed a bi-level model for a tradable credit scheme with equity constraints for the continuous network design problem. Grant-Muller and $\mathrm{Xu}$ [20] provided a complete review of the role of tradable credit schemes in road traffic congestion management. He et al. [21] studied tradable credit schemes on networks with different types of users.

With the purposes of mitigating congestion and maintain a reasonable revenue to the investors, a hybrid management scheme was proposed to improve the performance of the public-private mixed network. The entire network is divided into two sub-networks, namely, the public sub-network and the private sub-network. In the public sub-network, a tradable credit scheme is applied to mitigate traffic congestion by optimizing the credit charging levels and locations.
Meanwhile, in the private sub-network, the government franchises private firms to collect tolls, but toll levels and locations are determined by the government with the aim of alleviating traffic congestion. Meanwhile, the government guarantees private firms to obtain acceptable revenues. The management problem is formulated as a bi-level optimization structure as in [22]. The upper level model minimizes the total travel cost, considering toll/credit charging levels and locations as decision variables. The revenue threshold and the numbers of toll and credit charging links are incorporated as constraints into the upper level model. The lower level model describes the user equilibrium (UE) behaviour of travellers, given the tolls and credits transferred from the upper level.

Due to the complicated structure of the bi-level model, it is very difficult to obtain a global optimum of the problem with gradient-based methods. Then the bi-level optimization model is transformed into a single-level problem, by replacing the lower level user equilibrium (UE) program with its equivalent first-order conditions. Motivated by two seminal works [23-24], the UE conditions, travel time functions and charge location constraints are replaced by a set of linear equations through introducing some mixed integer variables. Therefore, the bi-level model is eventually transformed into a mixed integer linear program (MILP). Thus global optimum of the mixed management problem can be obtained by employing a commercial computing package, such as CPLEX.

The remaining part of the paper is organized as follows. In the following Section 2, we introduce the bi-level formulation of joint implementation of tradable credits and link tolls. In Section 3, the bi-level model is transferred into a single-level optimization problem, namely, a mixed integer linear program. Numerical examples are given in Section 4 and conclusions are provided in Section 5.

\section{BI-LEVEL FORMULATION OF TOLL AND TRADABLE CREDIT MIXED SCHEME}

Let $G(N, A)$ be a typical public-private mixed road network defined by a set $N$ of nodes and a set $A$ of directed links. The network contains public roads and private roads. Set $A_{1}$ denotes the link set of public roads, and set $A_{2}$ denotes the link set of the private roads, $A=A_{1} \cup A_{2}$. Let $W$ denote the set of origin-destination (O-D) pairs, and $R_{W}$ be the set of paths connecting $0-D$ pair $w, w \in W$. Let $v_{a}$ be the flow on the link $a \in A$, and $t_{a}\left(v_{a}\right)$ be the travel time on link $a \in A$, which is a continuous, convex and strictly increasing function of the link flow $v_{a}$. Let $\mathbf{v}=\left(v_{a}, a \in A\right)$ denote the vector of link flow. 
In this study, the management authority attempts to improve the network performance by simultaneously implementing a tradable credit scheme in the public sub-network and a toll charging scheme in the private sub-network.

Let $p$ denote the unit price of credit per unit, and $\mathbf{k}=\left(k_{a}, a \in A_{1}\right)$ denote the credit allocation scheme, where $k_{a}$ is the credit to be charged on link $a, a \in A_{1}$. Let $(\mathbf{k}, K)$ denote a credit scheme $\mathbf{k}$ with total amount of credit $K$. Let $\tau_{p r}=\left(\tau_{a}, a \in A_{2}\right)$ denote the toll set in the private sub-network, where $\tau_{a}$ is the toll level on link $a, a \in A_{2}$. We introduce two binary variables $\sigma_{a, b}$ and $\sigma_{a, c}$, to indicate whether a link is selected for toll/credit candidates or not. Here $\sigma_{a, c}$ is used to indicate whether link $a, a \in A_{1}$ is selected to collect mobility credit. If $\sigma_{a, c}=1$, link $a$ is the candidate link of credit charge, otherwise $\sigma_{a, c}=0$. Similarly, $\sigma_{a, b}$ is used to indicate whether link $a, a \in A_{2}$ is a candidate location for toll charge. If $\sigma_{a, b}=1$, link $a$ is a candidate toll link, otherwise $\sigma_{a, b}=0$.

It is naturally assumed that travellers follow a rational route choice behaviour of user equilibrium; namely, no one can reduce their travel cost by unilaterally changing the route. Given a mixed toll and credit charging scheme $\left(\mathbf{k}, K ; \tau_{p r}\right)$, the user equilibrium (UE) problem can be formulated as follows

$\min _{v} \sum_{a \in A} \int_{0}^{v_{a}}\left(t_{a}(\omega)+\sigma_{a, b} \tau_{a} \delta_{a, b}\right) d \omega$

subject to

$v_{a}=\sum_{w \in W} \sum_{r \in R} f_{r, w} \delta_{a, r}^{w}, \quad \forall a \in A$

$\sum_{r \in R} f_{r, w}=q_{w}, \quad \forall w \in W$

$f_{r, w} \geq 0, \quad \forall w \in W$

$\sum_{a \in A} \sigma_{a . c} k_{a} v_{a} \delta_{a, c} \leq K$

where $\delta_{a, c}$ and $\delta_{a, b}$ are two binary indicators. If link $a$ belongs to the set of public roads, i.e. $a \in A_{1}$, then $\delta_{a, c}=1$ and $\delta_{a, b}=0$. If link a belongs to the set of private roads, i.e. $a \in A_{2}$, then $\delta_{a, c}=0$ and $\delta_{a, b}=1$. Symbol $f_{r, w}$ represents the traffic flow on route $r \in R_{w}, w \in W$. Link-path incidence indicator $\delta_{a, r}^{w}$ equals 1 if route $r$ between $0-D$ pair $w \in W$ uses link $a \in A$, and 0 otherwise. Constraints 2 and 3 are the link-path incidence and demand conservation conditions, respectively. Constraint 5 ensures the feasibility of tradable credit scheme (i.e., the amount of collected credit cannot exceed the amount of issued credit).

The UE model can be rewritten as a set of equivalent complementary constraints

$$
\begin{aligned}
& {\left[\sum_{a \in A}\left(t_{a}\left(v_{a}\right)+\sigma_{a, b} \tau_{a} \delta_{a, b}+\sigma_{a, c} k_{a} p \delta_{a, c}\right) \delta_{a, r}-u_{w}\right] f_{r . W}} \\
& =0, \forall r, w
\end{aligned}
$$

$$
\begin{aligned}
& \sum_{a \in A}\left(t_{a}\left(v_{a}\right)+\sigma_{a, b} \tau_{a} \delta_{a, b}+\sigma_{a, c} k_{a} p \delta_{a, c}\right) \delta_{a, r}-u_{w} \\
& \geq 0, \forall r, w \\
& \left(K-\sum_{a \in A} \sigma_{a, c} k_{a} v_{a} \delta_{a, c}\right) p=0 \\
& K-\sum_{a \in A} \sigma_{a, c} k_{a} v_{a} \delta_{a, c} \geq 0 \\
& k_{a}\left(1-\delta_{a, c}\right)=0 \\
& \tau_{a}\left(1-\delta_{a, b}\right)=0 \\
& \sigma_{a, b}, \sigma_{a, c}, p \in\{0,1\}
\end{aligned}
$$

where $u_{w}$ represents the minimal travel cost of O-D pair $w \in W$. Without loss of generality, we assume the unit price of credit $p \in[0,1]$. According to the Conditions 8 and 9 , if there is a surplus of credit, the credits have no market value; namely, the price is zero.

Now the upper level optimization model is introduced. The management authority wants to minimize the total travel time in the network by implementing joint toll and credit scheme (simultaneously setting credit/toll levels and determining tolling locations). In addition, the number of toll /credit links is restricted, and profitable revenue for the private firm should be guaranteed. Therefore, the upper level model can be formulated as follows

$$
\begin{aligned}
& \min _{\sigma_{a, c}, \sigma_{a, b}, k_{a}, \tau_{a}} \sum_{a \in A}(\left(\sigma_{a, c}, \sigma_{a, b}, k_{a}, K, \tau_{a}\right) t_{a} \\
&\left(v_{a}\left(\sigma_{a, c}, \sigma_{a, b}, k_{a}, K, \tau_{a}\right)\right)
\end{aligned}
$$

subject to

$$
\begin{aligned}
& \sum_{a \in A_{1}} \sigma_{a, c} \leq N_{c} \\
& \sum_{a \in A_{2}} \sigma_{a, b} \leq N_{b} \\
& \sum_{a \in A_{2}} \sigma_{a, b} \tau_{a} V_{a} \geq P
\end{aligned}
$$

where $\mathrm{v}_{a}\left(\sigma_{a, c}, \sigma_{a, b}, k_{a}, K, \tau_{a}\right)$ is the solution of the UE problem. $N_{c}$ is the maximal number of public road links that are allowed to implement tradable credit scheme. $N_{b}$ is the maximal number of private road links that are permitted to be tolled. $P$ is the revenue threshold for private firm, which depends on the franchise contract. Constraints 14-16 give the three practical requirements.

\section{EQUIVALENT SINGLE-LEVEL MIXED INTEGER LINEAR PROGRAM}

Due to the complicated structure of the aforementioned bi-level model in Section 2, it is very difficult to obtain a global optimum solution with gradient-based methods. Therefore, in the section, a global solution algorithm is proposed by transforming the non-linear bi-level program into an equivalent single-level mixed integer linear program (MILP). The transformation 
includes linearization of the design objective function, link travel time function and the involved non-linear constraints.

\subsection{The linearization of travel time function}

In the study, the typical BPR function is used to define the link travel time as below:

$$
t_{a}\left(v_{a}\right)=t_{a}^{0}\left(1-\alpha\left(\frac{v_{a}}{c_{a}}\right)^{\beta}\right), \forall a \in A
$$

wheret $t_{a}^{0}$ denotes the free flow travel time and $c_{a}$ is the link capacity. Further, it is explained how to linearize the travel time function. $\alpha$ and $\beta$ are the parameters in the BPR function.

It is not difficult to see that the non-linear travel time function is with respect to decision variable $v_{a}$. Following Wang and Lo [23], we approximate the link travel time function by a set of piecewise linear functions. Let $\left[v_{a}, \bar{v}_{a}\right]$ be the feasible domain of link flow $v_{a}$. We then partition the domain into $M$ segments, where $M$ is a sufficiently large integer. Without loss of generality, the partitioned segments are ordered as $\underline{v}_{a}<g_{a, n}<g_{a, n+1}<\bar{v}_{a}, n=1, \ldots, M-1$. It follows from the first-order Taylor series that, for each feasible region $\left[g_{a, n}, g_{a, n+1}\right]$, we can obtain the following approximation for the link travel time function

$$
\begin{aligned}
& t_{a}\left(v_{a}\right)=t_{a}^{0}\left(1+\alpha\left(\frac{v_{a}}{c_{a}}\right)^{\beta}\right) \Rightarrow \\
& \Rightarrow\left\{\begin{array}{l}
L \xi_{a, n} \leq v_{a}-g_{a, n} \leq U\left(1-\xi_{a, n}\right)-\varepsilon \\
\xi_{a, n} \in\{0,1\} \\
L_{a, n}=\xi_{a, n+1}-\xi_{a, n} \\
L\left(1-\lambda_{a, n}\right) \leq t_{a}\left(a_{n}^{a} v_{a}+b_{n}^{a}\right) \leq U\left(1-\lambda_{a, n}\right)
\end{array}\right.
\end{aligned}
$$

where $L$ is a very large negative constant and $U$ is a very large positive constant; $\varepsilon$ is a very small positive constant; $\lambda_{a, n}, \xi_{a, n}$ are the auxiliary variables that help determine the feasible domain of $v_{a} \cdot a_{n}^{a}$ and $b_{n}^{a}$ are coefficients to be determined. Accordingly, coefficient $a_{n}^{a}$ is determined by derivatives of the travel time function with respect to $v_{a}$ at $g_{a, n}$

$a_{n}^{a}=\frac{\partial t_{a}}{\partial v_{a}} \mid g_{a, n}=\frac{\alpha \beta t_{a}^{0} g_{a, n}^{\beta-1}}{c_{a}^{\beta}}$

Similarly, coefficient $b_{n}^{a}$ can be given by

$$
\begin{aligned}
b_{n}^{a} & =t_{a}\left(g_{a, n}\right)-g_{a, n} \cdot \frac{\partial t_{a}}{\partial v_{a}} \mid g_{a, n}= \\
& =t_{a}^{0}\left(1+\alpha\left(\frac{g_{a, n}}{c_{a}}\right)^{\beta}\right)-\frac{\alpha \beta t_{a}^{0} g_{a, n}{ }^{\beta}}{c_{a}^{\beta}}
\end{aligned}
$$

\subsection{Linearization of the non-linear objective function}

Let $s_{a}\left(v_{a}\right)=t_{a}\left(v_{a}\right) v_{a}$. Then, the objective function in the upper level can be rewritten as: $\min \sum_{a \in A} s_{a}\left(v_{a}\right)=\min \sum_{a \in A} v_{a} t_{a}^{0}\left(1+\alpha\left(\frac{v_{a}}{c_{a}}\right)^{\beta}\right)$

A similar method used in Section 3.1 can be applied. Thus, we get the approximation:

$$
\begin{aligned}
& \mathrm{s}_{a}\left(v_{a}\right)=v_{a} t_{a}^{0}\left(1+\alpha\left(\frac{v_{a}}{C_{a}}\right)^{\beta}\right) \Rightarrow \\
& \Rightarrow\left\{\begin{array}{l}
L \xi_{a, n} \leq v_{a}-g_{a, n} \leq U\left(1-\xi_{a, n}\right)-\varepsilon \\
\xi_{a, n} \in\{0,1\} \\
\lambda_{a, n}=\xi_{a, n+1}-\xi_{a, n} \\
L\left(1-\lambda_{a, n}\right) \leq s_{a}\left(I_{n}^{a} v_{a}+z_{n}^{a}\right) \leq U\left(1-\lambda_{a, n}\right)
\end{array}\right.
\end{aligned}
$$

where coefficient $I_{n}^{a}$ is determined by derivatives of function $s_{a}\left(v_{a}\right)$ with respect to $v_{a}$ at $g_{a, n}$ :

$I_{n}^{a}=\left.\frac{\partial s_{a}}{\partial v_{a}}\right|_{g_{a, n}}=t_{a}^{0}+\frac{\alpha(\beta+1) t_{a}^{0} g_{a, n}{ }^{\beta}}{c^{\beta}}$

and coefficient $z_{n}^{a}$ is given by:

$$
\begin{aligned}
z_{n}^{a}= & s_{a}\left(g_{a, n}\right)-g_{a, n} \cdot \frac{\partial t_{a}}{\partial v_{a}} \mid g_{a, n} \\
= & g_{a, n} t_{a}^{0}\left(1+\alpha\left(\frac{g_{a, n}}{C_{a}}\right)^{\beta}\right)- \\
& -g_{a, n}\left(t_{a}^{0}+\alpha(\beta+1) t_{a}^{0}\left(\frac{g_{a, n}}{C_{a}}\right)^{\beta}\right)
\end{aligned}
$$

So far, we have finished the linearization of the objective function.

\subsection{Linearization of constraints}

In the developed bi-level optimization model, Constraints 14 and 15 are linear, but Constraint 16 is non-linear. The same linearization method can be applied to transform the non-linear Constraint 16 into equivalent linear conditions by introducing a set of binary variables. As for the user equilibrium constraint in the lower level, it can be replaced by the first order conditions; namely, a set of non-linear complementary conditions. Fortunately, they can be transformed into equivalent linear constraints by incorporating a set of integer variables; the detailed procedure and formulations can be found in Wang and Lo [23] and Zhang and van Wee [22]. To save space, we do not display the entire linearized formulation of the lower level program. The reformulated MILP model can be efficiently solved by some commercial solvers, e.g., CPLEX and GUROBI.

\section{NUMERICAL EXAMPLES}

In this section, some numerical experiments are carried out to test the model and hopefully to obtain some insightful implications. More specifically, we want to see the varying patterns of the system travel cost against the revenue threshold, and the number of toll roads. The used transportation network is depicted in Figure 1. The network consists of 13 nodes and 19 links. Four OD pairs are considered. The travel 
demands of these OD pairs are given in Table 1 . The free flow travel times and the capacities of the links are provided in Table 2. Links 1, 6, 8-10, 12-13 and 17 are private roads (solid lines), and others are public roads (dash-dot lines). And we evaluate the parameters $\alpha=0.15, \beta=4$ that were used in [22]. The traditional traffic assignment shows that the total travel time of the network is 5.850105 at user equilibrium and $5.725 \cdot 10^{5}$ at system optimum. To analyse the effects of the hybrid management policy proposed in the paper, the commercial optimization software IBMILOG-CPLEX-12.6 is utilized to solve the reformulated mixed integer linear program. The following three experiments are carried out.

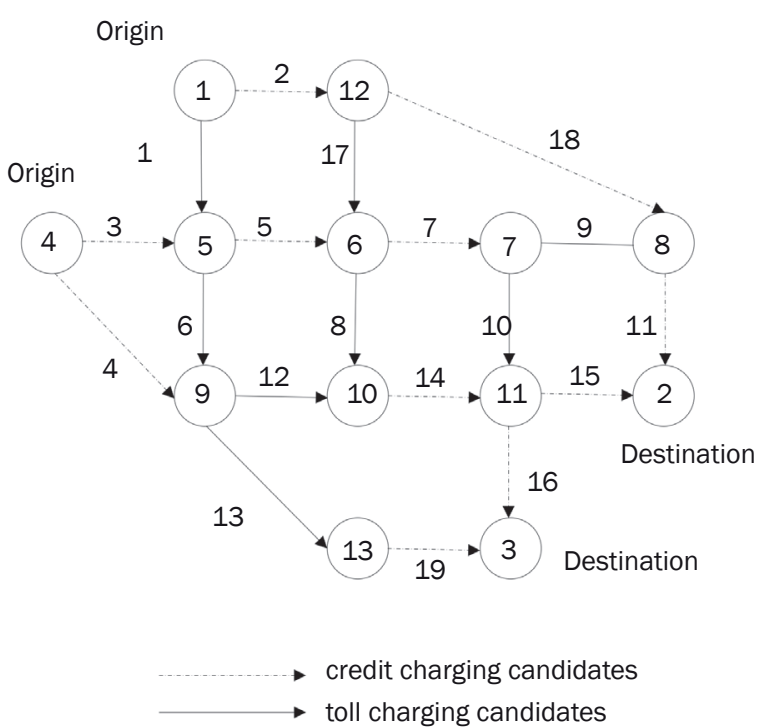

Figure 1 - A thirteen-node transportation network.

Table 1 - Travel demands for the OD pairs

\begin{tabular}{|c|c|c|c|c||}
\hline OD pair & $1 \rightarrow 2$ & $1 \rightarrow 3$ & $4 \rightarrow 2$ & $4 \rightarrow 3$ \\
\hline \hline Demand & 4,000 & 4,000 & 4,000 & 4,000 \\
\hline
\end{tabular}

\section{Experiment $A$}

In the experiment, we demonstrate the performance of our model and discuss the management effects of the mixed charging scheme by varying the number of toll roads. All public roads are assumed to be considered as candidate locations of credit charge. The revenue threshold in Constraint 16 is set to be $P=0$, i.e., the private firm does not have a requirement on revenue.

Based on the bi-level model and its mixed integer linear reformulation, we compute the optimal toll levels and locations when the number of toll links is set to be $0,1,3,5$, and 8 , respectively. The results are shown in Figure 2 and Table 3, which provide the optimal toll locations and toll levels, as well as the corresponding total travel time of the whole network. When no link is subject to toll charge; namely, only the tradable credit scheme is applied; the total travel time of the network is $5.749 \cdot 10^{5}$, which is a substantial decrease compared with the UE situation. If only one link is allowed for toll charge, link 9 is the optimal toll location. The optimal toll is 51.80 , and the corresponding total travel time is $5.741 \cdot 10^{5}$ in the situation. As the number of toll links increases, total travel time of the network decreases. When five links are allowed for toll charge, the network total travel time decreases to the system optimum situation. Therefore, when the number of toll links increases to eight, although different solutions of toll locations and toll levels are obtained, the total travel time of the network cannot further decrease any longer. This experiment shows that only applying tradable credit scheme can have a very acceptable improvement in terms of system travel time reduction. Moreover, the system can be further improved by implementing toll charge, and the system optimum can be achieved by charging only a subset of private links.

\section{Experiment $B$}

The second experiment is used to study the effects of the hybrid management by simultaneously varying the numbers of toll links and credit links. We go over all the combinations of the numbers of toll and credit

Table 2 - Free-flow times and capacities of the links

\begin{tabular}{|c|c|c|c|c|c|c|c|c|c|c||}
\hline \hline Link & 1 & 2 & 3 & 4 & 5 & 6 & 7 & 8 & 9 & 10 \\
\hline \hline$t_{a}^{0}$ & 4.35 & 4.28 & 4.16 & 3.92 & 3.89 & 4.86 & 4.28 & 5.91 & 4.09 & 4.92 \\
\hline$c_{a}$ & 3,000 & 2,000 & 3,000 & 2,000 & 6,000 & 2,000 & 6,000 & 2,000 & 3,000 & 3,000 \\
\hline \hline Link & 11 & 12 & 13 & 14 & 15 & 16 & 17 & 18 & 19 & \\
\hline \hline$t_{a}^{0}$ & 4.29 & 5.62 & 4.21 & 4.26 & 5.73 & 4.31 & 2 & 4.36 & 4.89 & \\
\hline$c_{a}$ & 6,000 & 2,000 & 2,000 & 2,000 & 2,000 & 3,000 & 2,000 & 1,000 & 1,000 & \\
\hline
\end{tabular}




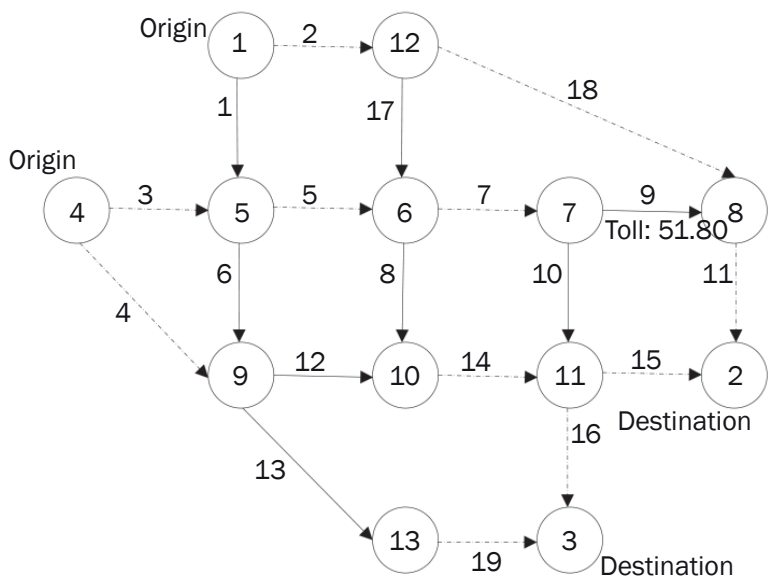

a) One toll link

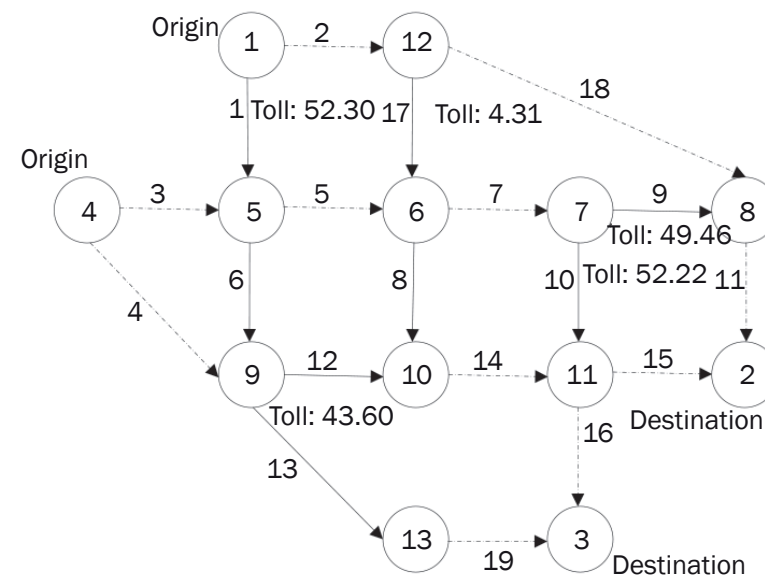

c) Five toll link

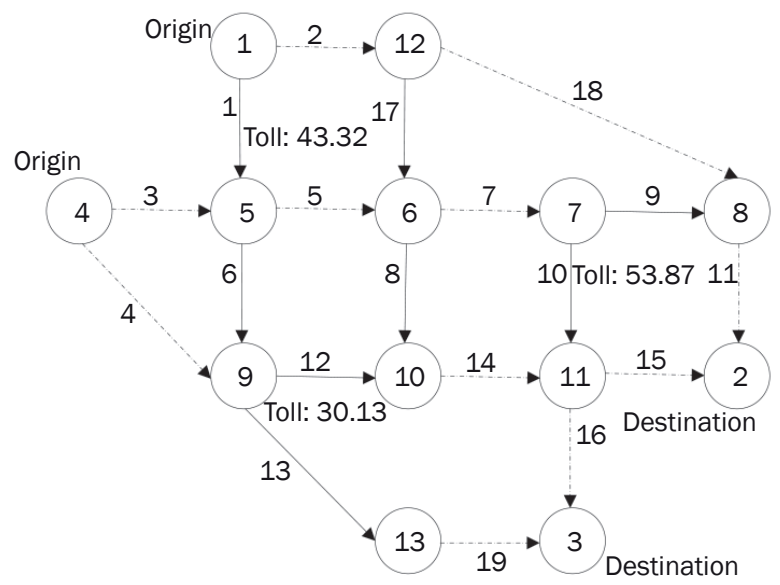

b) Three toll link

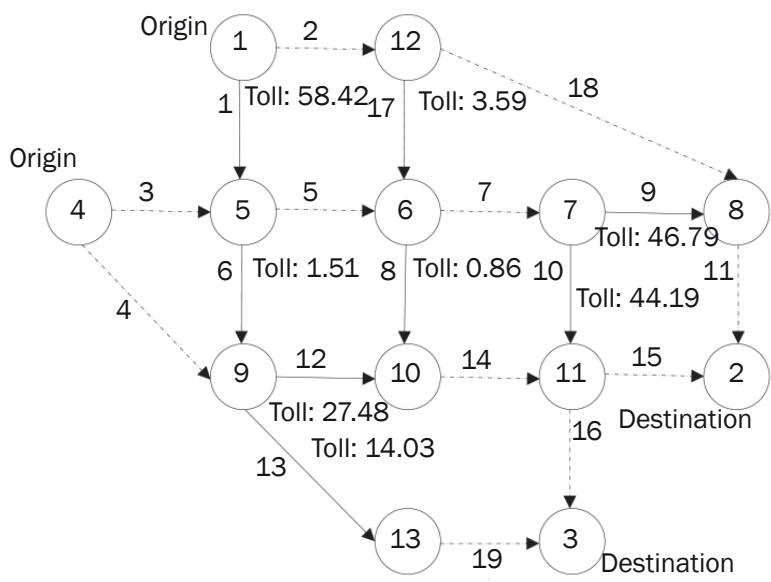

d) Nine toll link

Figure 2 - Results of the toll level and location

Table 3 - The results of the toll level and location

\begin{tabular}{|c|c|c|c|c|}
\hline \multirow{2}{*}{ Max no. of toll links } & \multirow{2}{*}{ Total travel time } & \multirow{2}{*}{ Optimal toll links } & \multicolumn{2}{|c|}{ Optimal tolls } \\
\hline & & & Link & Toll \\
\hline 0 & $5.749 \cdot 10^{5}$ & - & - & - \\
\hline 1 & $5.741 \cdot 10^{5}$ & 9 & 9 & 51.80 \\
\hline \multirow{3}{*}{3} & \multirow{3}{*}{$5.73 \cdot 10^{5}$} & \multirow{3}{*}{$1,10,12$} & 1 & 43.32 \\
\hline & & & 10 & 53.87 \\
\hline & & & 12 & 30.13 \\
\hline \multirow{5}{*}{5} & \multirow{5}{*}{$5.725 \cdot 10^{5}$} & \multirow{5}{*}{$1,9,10,12,17$} & 1 & 52.30 \\
\hline & & & 9 & 49.46 \\
\hline & & & 10 & 52.22 \\
\hline & & & 12 & 43.60 \\
\hline & & & 17 & 4.31 \\
\hline \multirow{8}{*}{8} & \multirow{8}{*}{$5.725 \cdot 10^{5}$} & \multirow{8}{*}{$1,6,8,9,10,12,13,17$} & 1 & 58.42 \\
\hline & & & 6 & 1.51 \\
\hline & & & 8 & 0.86 \\
\hline & & & 9 & 46.79 \\
\hline & & & 10 & 44.19 \\
\hline & & & 12 & 27.48 \\
\hline & & & 13 & 14.03 \\
\hline & & & 17 & 3.59 \\
\hline
\end{tabular}


links, and obtain the social travel time of each combination, respectively. The revenue threshold in Constraint 16 is set to be as in the previous experiment.

The results are shown in Figure 3. Firstly, we consider two extreme cases. If the numbers of toll and credit links are both 0 , namely in the UE situation, the total travel time is $5.850 \cdot 10^{5}$. On the contrary, if the hybrid management is implemented in the entire 8 toll links and 11 credit links, a minimum system travel time equivalent to the first-best system optimum situation can be achieved, i.e. the total travel time is $5.725 \cdot 10^{5}$. The good news is that the system optimum can also be achieved with fewer toll links and credit links. As depicted in Figure 3, the system optimum can be obtained when the combinations of the numbers of toll links and credit links are $(8,9),(8,10),(8,11),(7,9)$, $(7,10),(7,11),(6,9),(6,10),(6,11),(5,10)$, and $(5,11)$ respectively. Furthermore, in the hybrid management scheme, total travel time is more sensitive to the number of credit links than that of toll links.

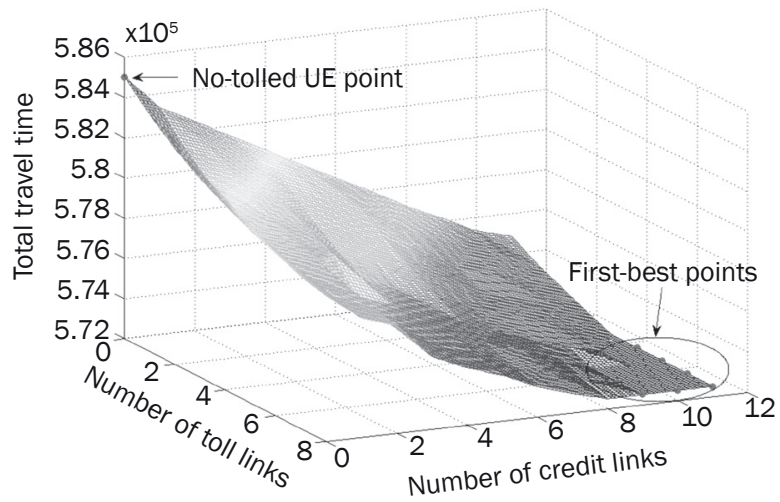

Figure 3 - Variation of system travel time with different toll links and credit links

\section{Experiment $\mathrm{C}$}

The third example is to show the influence of revenue requirement on the system travel time. Four situations have been set, when the combinations of numbers of toll links and credit links are $(1,11),(1,0)$, $(8,11)$, and $(8,0)$, respectively. In the four situations, we examine the relationship between revenue requirement and total travel time. The results are shown in Figure 4. The results show that total travel time can only be reduced by decreasing the requirement of revenue.

When the number of credit link is 0 and the number of toll link is 1 , the total travel time is always maximal in the four situations. Furthermore, in the situation, the total travel time increases remarkably with the increasing revenue requirement.

When the number of credit link is 11 and the number of toll link is 8 , the total travel time is always minimal in the four situations. In the situation, the required revenue is less than $2 \cdot 10^{5}$ for system optimum. The total travel time increases slowly when the revenue requirement is higher than $2 \cdot 10^{5}$.
The other two cases are in between the aforementioned two situations. As the required revenue increases, the increasing speed of the system travel time is also between the two aforementioned situations. By comparing the four situations, it can be clearly seen that the sensitivity of revenue to total travel time is negatively related with the number of the toll and credit links. In other words, when the number of toll and credit links is small, the system travel time increases rapidly with the required revenue. With more toll links and credit links, the system travel time increases much slower with the increase of required revenue.

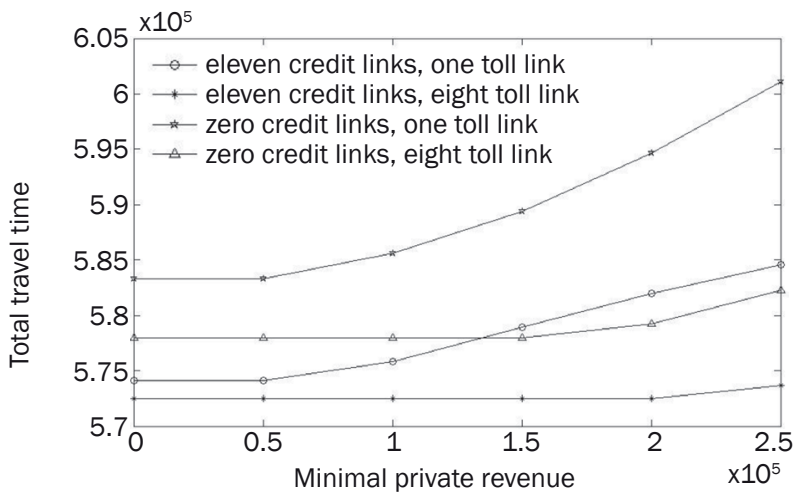

Figure 4 - Relationship between private revenue and total travel time

\section{CONCLUSIONS}

The paper investigates a hybrid management policy of road tolls and tradable credits in mixed road networks with both public and private roads. The entire network is divided into two sub-networks, namely, the public sub-network and the private sub-network. In the public sub-network, a tradable credit scheme is applied to mitigate traffic congestion. In the private sub-network, tolls are collected by the private company, but the toll levels and toll locations are determined by the government. The purpose of toll charge is twofold, on one hand, the government uses it as a tool of mitigating congestion, on the other hand, a threshold of revenue should be guaranteed for the profitability of the private company.

Due to the complicated structure of the bi-level model, it is very difficult to obtain a global optimum of the problem with gradient-based methods. Then the bi-level optimization model is transformed into a single-level problem, by replacing the lower level user equilibrium (UE) program with its equivalent first-order conditions. Motivated by two seminal works, the UE conditions, travel time functions and charge location constraints are replaced by a set of linear equations through introducing some mixed integer variables. Therefore, the bi-level model is eventually transformed into a mixed integer linear program (MILP) 
that can be solved with commercial software easily. Numerical examples are provided to demonstrate the effectiveness of the developed model and the efficiency of the proposed algorithm. It is shown that the mixed management schemes can achieve favourable targets; namely, joint implementation of road tolls and tradable credits can effectively mitigate traffic congestion and meanwhile maintain reasonable revenue for the private company. This experiment also shows that only applying tradable credit scheme can have a very acceptable improvement in terms of system travel time reduction. Moreover, the system can be further improved by implementing toll charge, and system optimum can be achieved by charging only a subset of private links. Furthermore, in the hybrid management scheme, the total travel time is more sensitive to the number of credit links than that of toll links. By comparing various situations, we can see that the sensitivity of revenue to total travel time is negatively related with the number of the toll and credit links.

In the paper, the purpose of management is to mitigate congestion, namely, by minimizing the system travel time, which can be seen in the upper-level models. Whether system optimum can be achieved or not, depends really on the structure of the private sub-network and public sub-network, and more importantly it depends on the candidate sets of toll and credit links. If all links in the public sub-network are subject to credit charge and all links in the private sub-network are subject to toll charge, a system optimum is guaranteed.

\section{ACKNOWLEDGEMENTS}

This study has been substantially supported by several grants from the National Natural Science Foundation Council of China (Project No. 71531011, 71361130014, 71471134, 71601142), and a grant from the Shanghai Shuguang Program (Project \#13SG23)

江楠, 博士生 1 (通讯作者)

电子邮箱： jiangnantju@126. com

张小宁，博士 2

电子邮箱：cexzhang@tongji.edu.cn

王华，博士 2

电子邮箱: hwang63@tongji. edu. cn

1 同济大学道路与交通工程教育部重点实验室,

中华人民共和国, 上海市，曹安公路4800号 (邮编： 201804)

2 同济大学经济与管理学院,

中华人民共和国, 上海市，四平路1239号 (邮编：200092)

\section{基于道路收费与可交易路票同步优化方法的公有-私 营混合路网管理模型}

摘要

本文采用道路收费和可交易路票的混合优化方法运用 于管理公有-私营混合路网。其中，可交易路票被用于在 混合路网的公有子路网管理。同时为了兼顾缓解拥挤和保
证私营企业利润两个目标, 混合路网的私有子路网采用道 路收费的方法进行管理, 而收费额度和路段由政府决定。 本文采用双层规划模型去描述这个优化管理问题，上层模 型的目标为最小化总出行时间, 约束为保证一定的私营企 业利润; 下层模型为用户均衡问题。为了获得全局最优 解, 我们将双层规划模型转化为利于优化软件求解的等价 单层混合整数线性规划。数值算例证明了所提出模型和算 法的有效性。算例还表明混合管理方案能够达到良好的管 理目标，即收费和可交易路票的同步优化方法在有效的缓 解拥挤的同时还能够保证私营企业获得合理的收入。

\section{REFERENCES}

[1] Beckmann MJ. On optimal tolls for highways, tunnels and bridges. In: Edie LC, Herman R, Rolhery R, eds. Vehicular traffic science: proceedings of the third international symposium on the theory of traffic flow. New York: American Elsevier; 1965. p. 331-341.

[2] Dafermos SC, Sparrow FT. Optimal Resource Allocation and Toll Patterns in User-Optimised Transport Networks. Journal of Transport Economics and Policy. 1971;5(2): 184-200.

[3] Dafermos SC. Toll Patterns for Multiclass-User Transportation Networks. Transportation Science. 1973;7(3): 211-23.

[4] Smith MJ. The marginal cost taxation of a transportation network. Transportation Research Part B. 1979;13(1): 237-242.

[5] Yang $\mathrm{H}$, Huang $\mathrm{H}$. Principle of marginal-cost pricing: how does it work in a general road network? Transportation Research Part A. 1998;32(1): 45-54.

[6] Verhoef ET, Nijkamp P, Rietveld P. Second-Best Congestion Pricing: The Case of an Untolled Alternative. Journal of Urban Economics. 1996;40(3): 279-302.

[7] Liu LN, McDonald JF. Economic efficiency of second-best congestion pricing schemes in urban highway systems. Transportation Research Part B. 1999;33(3): 157-88.

[8] Zhang $X$, Yang $H$. The optimal cordon-based network congestion pricing problem. Transportation Research Part B. 2004;38(6): 517-37.

[9] Sumalee A. Multi-concentric optimal charging cordon design. Transportmetrica. 2007;3(1): 41-71.

[10] Di X, Liu H, Ban X. Second best toll pricing within the framework of bounded rationality. Transportation Research Part B. 2016;83: 74-90.

[11] De Palma A. A Game-Theoretic Approach to the Analysis of Simple Congested Networks. The American Economic Review. 1992;82(2): 494-500.

[12] Yang H, Meng Q. Highway pricing and capacity choice in a road network under a build-operate-transfer scheme. Transportation Research Part A. 2000;34(3): 207-22.

[13] Yang H, Woo KK. Competition and equilibria of private toll roads in a traffic network. Transportation Research Record. 2011;1733: 15-22.

[14] Xiao F, Yang H, Han D. Competition and efficiency of private toll roads. Transportation Research Part B. 2007;41(3): 292-308.

[15] Wu D, Yin Y, Yang $H$. The independence of volume-capacity ratio of private toll roads in general networks. Transportation Research Part B. 2011;45(1): 96-101.

[16] Yang $H$, Wang $X$. Managing network mobility with tradable credits. Transportation Research Part B. 2011;45(3): 580-94.

[17] Xiao F, Qian Z, Zhang H. Managing bottleneck 
congestion with tradable credits. Transportation Research Part B. 2013;56:1-14.

[18] Nie Y. Transaction costs and tradable mobility credits. Transportation Research Part B. 2012;46: 189-203.

[19] Wang G, Gao ZY, Xu M, Sun H. Models and a relaxation algorithm for continuous network design problem with a tradable credit scheme and equity constraints. Computers \& Operation Research. 2014;41: 252-261.

[20] Grant-Muller S, Xu M. The Role of Tradable Credit Schemes in Road Traffic Congestion Management. Transport Reviews. 2014;34: 128-149.

[21] He F, Yin YF, Shirmohammadi N, Nie Y. Tradable credit schemes on networks with mixed equilibrium behaviors. Transportation Research Part B. 2013;57: 47-65.

[22] Zhang $X$, van Wee B. Enhancing transportation network capacity by congestion pricing with simultaneous toll location and toll level optimization. Engineering Optimization. 2011;44(4): 477-88.

[23] Wang DZW, Lo HK. Global optimum of the linearized network design problem with equilibrium flows. Transportation Research Part B. 2010;44(4): 482-92.

[24] Wang S, Meng Q, Yang H. Global optimization methods for the discrete network design problem. Transportation Research Part B. 2013;50: 42-60. 